\title{
Modeling on phosphor sedimentation phenomenon during curing process of high power LED packaging
}

Yiman Wang, Huai Zheng, Run Hu and Xiaobing Luo*

\footnotetext{
* Correspondence: luoxb@hust.edu.cn School of Energy and Power Engineering, Huazhong University of Science and Technology, Wuhan 430074, China
}

\begin{abstract}
In the LED packaging, the phosphor particles are usually mixed with silicone matrix material, and then dispensed onto the LED chips directly. Since the density of the phosphor particle is bigger than that of the silicone, the phosphor particles may settle in the silicone matrix because of the gravity during dispensing and curing processes. In this paper, a model was developed to investigate the phosphor sedimentation phenomenon in silicone during isothermal curing according to the Stokes' law, and the size distribution of phosphor particles and the viscosity-time function of silicone during curing were built for the model. We compared the modeling results with the experimental results and found that the model can well simulate the sedimentation phenomenon. The modeling results show that the sedimentation velocity is inversely proportional to the viscosity of silicone and proportional to the size of phosphor particle. Phosphor particles sedimentation lasts for about 5 minutes during isothermal curing at $85^{\circ} \mathrm{C}$, and the big particles accumulate in the bottom of the sample.

Keywords: Phosphor sedimentation; Isothermal curing; Modeling
\end{abstract}

\section{Background}

High power light-emitting diode (LED) has grown explosively in recent years for its extraordinary characteristics, such as high luminous efficiency, low power consumption, high reliability, and long life. Thus, LEDs are considered as the most potential solid-state light source for the next generation lighting and have been widely used in our daily lives [1-3].

So far, the most common approach for getting white LEDs is based on mixing blue lights emitted from LED chip with yellow lights reemitted from phosphor material (as shown in Figure 1).

In the LED packaging shown in Figure 1, phosphor particles are usually mixed with silicone matrix material, and then dispensed onto LED chips directly [4]. Since the arrangement of phosphor particles plays an important role in determining the optical performances of white LEDs, the concentration of phosphor particles should be as uniform as possible. However, the density of phosphor particles (about $4800 \mathrm{~kg} / \mathrm{m}^{3}$ ) is bigger than that of silicone (about $1120 \mathrm{~kg} / \mathrm{m}^{3}$ ). Phosphor particles would settle in silicone matrix because of the gravity during dispensing and curing processes as a 


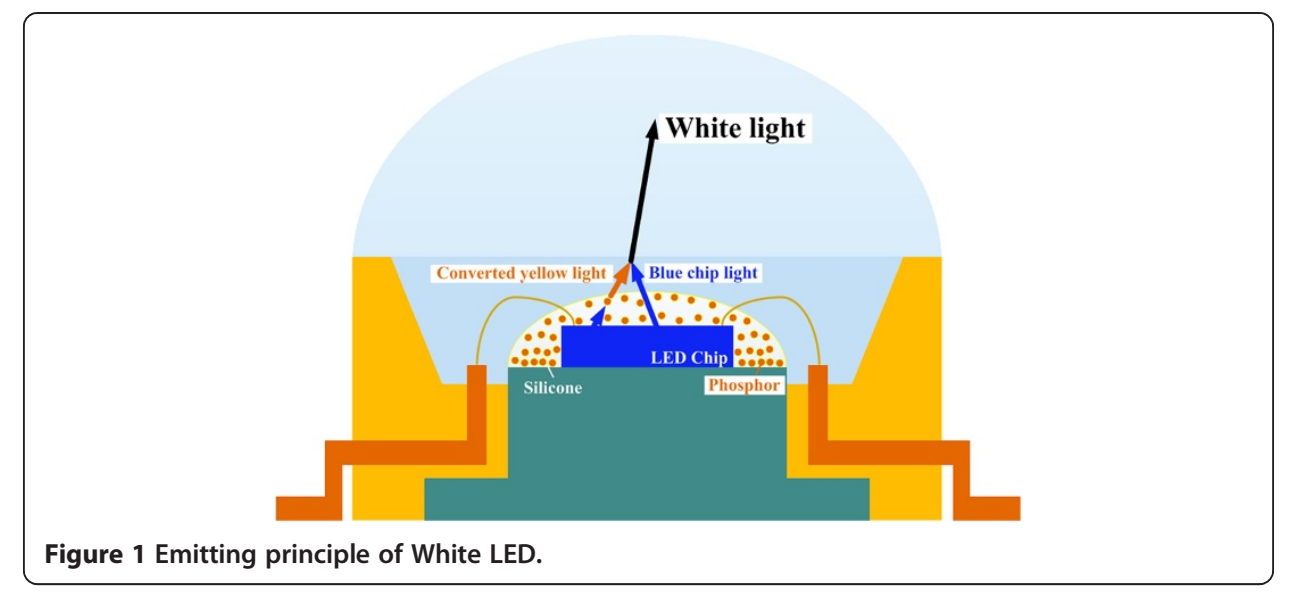

consequence. With the settling of phosphor particles, the luminous efficiency would decrease and so did the homogeneity of the color. Thus, it is a serious problem in the LED packaging.

In recent years, few experiments and simulation studies have been performed to investigate phosphor sedimentation phenomenon. Sommer et al. [5] simulated phosphor sedimentation using the commercial software package ASAP (Advanced System Analysis Program). They found that LED optical performances, such as the color temperature and the flux output, are highly dependent on phosphor distribution. Lee et al. [6] investigated the influence of phosphor sedimentation on the white LEDs with different structure chips, and found that phosphor sedimentation cause $20 \%$ difference in the luminous efficiency of white LEDs. Hu et al. [7] established a multi-layer phosphor model by assuming the concentration gradient of phosphor, which was applied to study the effect of phosphor sedimentation on the light extraction efficiency. They found that phosphor sedimentation caused 13.04\% drop of light extraction efficiency.

The above researches focused on studying the influence of phosphor sedimentation on the performance of white LEDs, while the investigations about phosphor sedimentation during curing process of white LED packaging have not been found yet. In this study, phosphor sedimentation during curing process was investigated by modeling according to the Stokes' law. In order to validate the accuracy of the model, longitudinal cross-sectional microstructure photograph of a sample was acquired using the scanning electron microscope (SEM). The comparison between modeling results and experimental results proves that the model can well predict this sedimentation process.

\section{Methods}

\section{Forces analysis}

The Reynolds number for a spherical particle moving through a viscous fluid is as follows:

$$
\operatorname{Re}=2 \rho_{f} v r / \eta,
$$

where $\rho_{f}$ is the density of the viscous fluid, $v$ is the velocity of the particle, $r$ is the radius of the particle, and $\eta$ is the viscosity of the viscous fluid. Figure 2 shows the microstructure photograph of the phosphor particles. Since the radius of the phosphor 


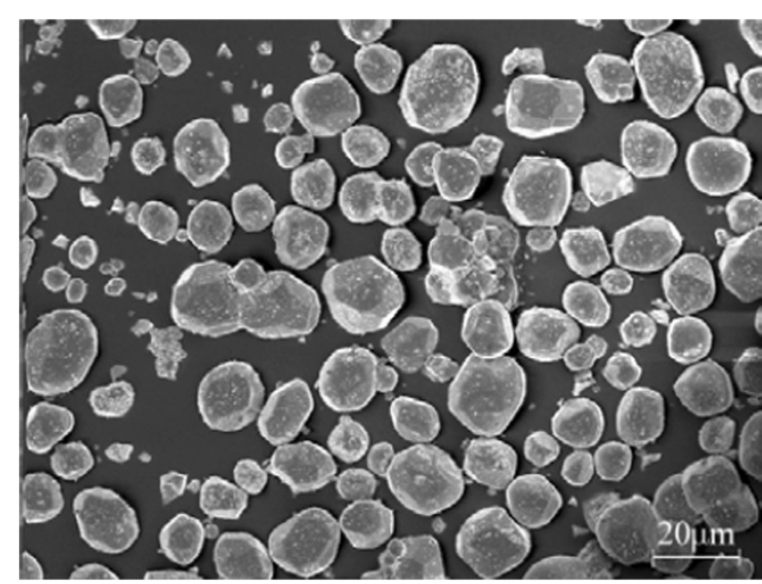

Figure 2 Microstructure photograph of phosphor particles.

particle is at micron level and silicone is a high-viscosity fluid, the Reynolds number for phosphor particle sedimentation in silicone is quite small, so the inertia force is much smaller than the resistance force, which means the inertia force can be ignored.

According to the Stokes' law, the resistance force for a spherical particle moving through a viscous fluid can be written as follows, ignoring the inertia force [8]:

$$
F_{\text {Stokes }}=6 \pi \eta r \nu
$$

Figure 3 shows the forces acting on the phosphor particle. Taking the gravitational and the buoyancy forces into account, $F_{\text {grav }}$ is written as follows:

$$
F_{\text {grav }}=(4 / 3) \pi r^{3} g\left(\rho_{p}-\rho_{f}\right)
$$

where $g$ is the acceleration of gravity, and $\rho_{p}$ is the density of the particle.

Hence, the kinetic equation for the phosphor particle sedimentation in the silicone is as follows:

$$
F_{\text {grav }}-F_{\text {stokes }}=m a=(4 / 3) \pi r^{3} \rho_{p} \frac{d v}{d t}
$$

Substituting Eq. (2) and Eq. (3) into Eq. (4), and the velocity of the particles could be described as this:

$$
v(t)=\left(A-A e^{-B t / C}\right) / B
$$

where $A=(4 / 3) \pi r^{3} g\left(\rho_{p}-\rho_{f}\right), B=6 \pi \eta r$, and $C=(4 / 3) \pi r^{3} \rho_{p}$. Thus, the size distribution of the phosphor particles and the viscosity-time function of silicone during curing are the key to get the velocity of any phosphor particle at any time during the sedimentation process, according to $\mathrm{A}, \mathrm{B}$ and $\mathrm{C}$. 


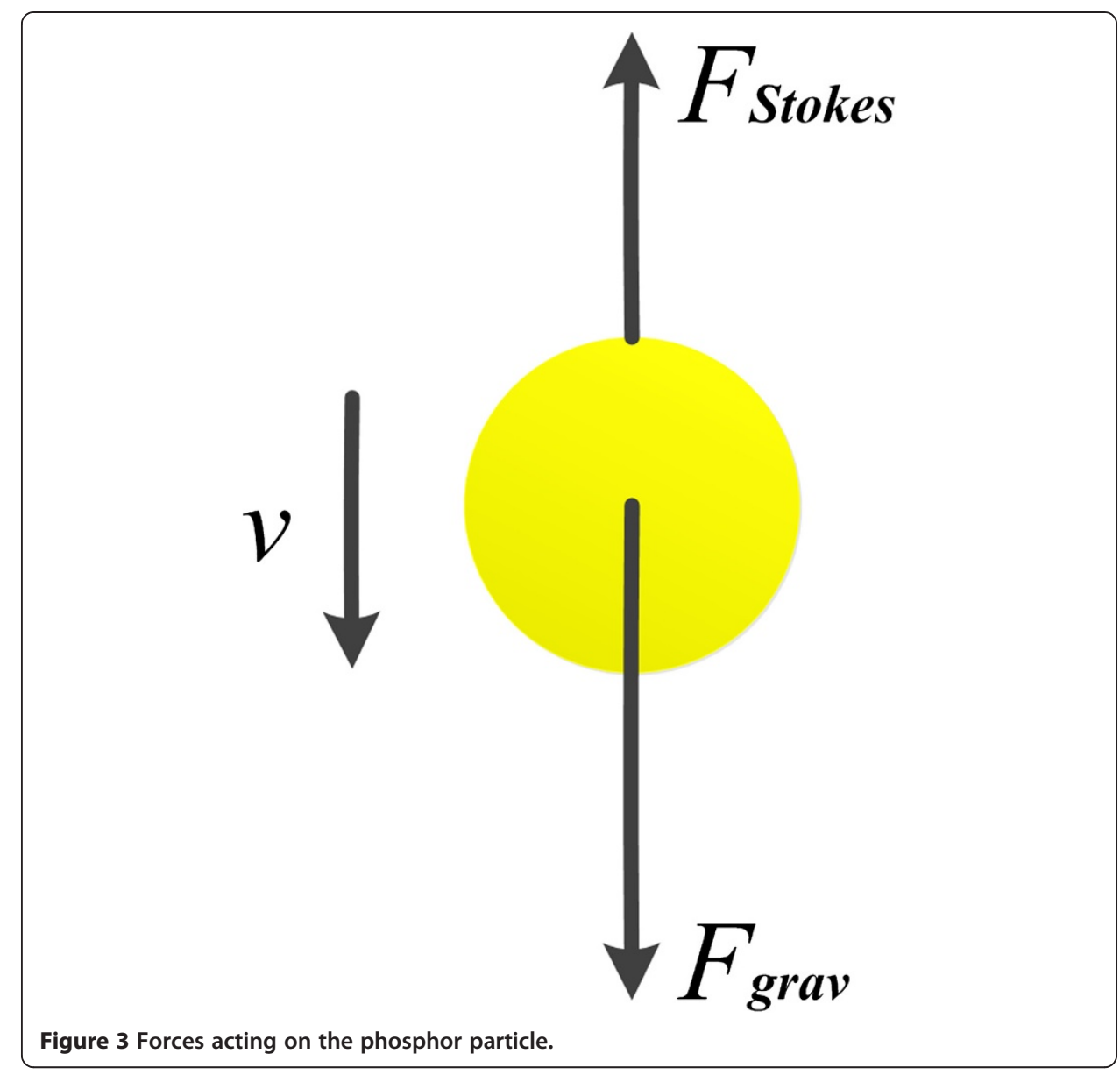

\section{Size distribution of phosphor}

Figure 4 shows the size distribution of phosphor particles provided by the phosphor manufacturer named Intematix. Fitting the distribution curve of the particles gives the size distribution function as follows:

$$
f(x)=\left\{\begin{array}{lr}
0.124 e^{0.391 x}-1.040 & x<12.5 \\
-56.741+5.802 x & 12.5 \leq x<21.2, \\
-504.968+27.245\left(1-e^{-0.041 x}\right)+577.003\left(1-e^{-0.152 x}\right) & x \geq 21.2
\end{array}\right.
$$

where $f(x)$ means the probability that the particle size is smaller than $x$.

\section{Viscosity-time function of silicone}

In order to find the variation of viscosity with time during isothermal curing, CPA2000+ viscometer was used. Keeping the temperature of the heating plate at $85^{\circ} \mathrm{C}$, a series of data was measured with the lapse of time. Figure 5 shows the relationship between the viscosity of silicone and time during isothermal curing at $85^{\circ} \mathrm{C}$. The viscosity increases exponentially with time [9]. Fitting the curve gives the viscosity-time function as follows:

$$
\eta(t)=0.131+e^{0.024 t-3.587}
$$

Substituting Eq. (6) and Eq. (7) into Eq. (5), the velocity of any phosphor particle at any time during the sedimentation is obtained, and the displacement of the particle can be calculated by integration as a result. 


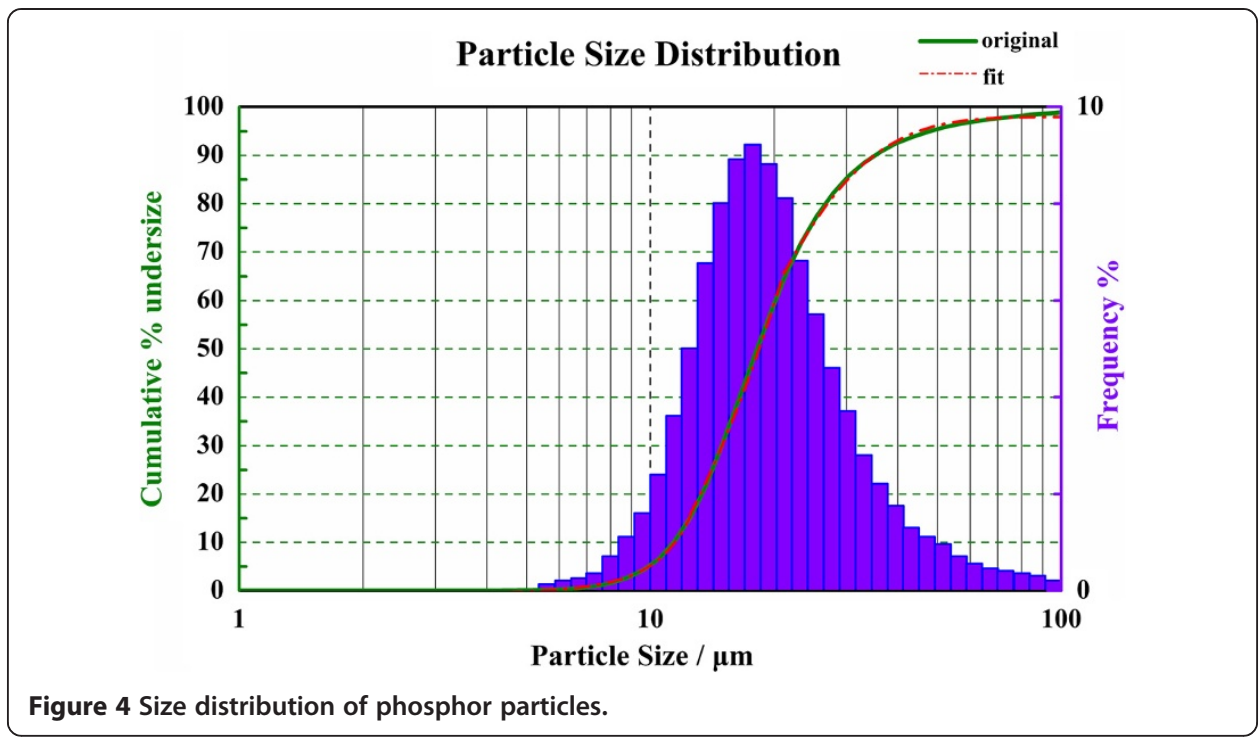

\section{Experiment}

Experiment has been carried out to verify the accuracy of the model. Silicone matrix mixed with $16.7 \mathrm{wt} . \%$ phosphor was used in our experiment. The silicone material is produced by Dow Corning, and the model number is OE-6550. Figure 6 shows the processes of the experiment. In Figure 6(a), phosphor slurry was dispensed into a mold ( $5 \mathrm{~mm} * 2 \mathrm{~mm} * 1.2 \mathrm{~mm}$ ) using a capillary. Then the mold filled with phosphor slurry was placed on a heating plate and heat-cured at $85^{\circ} \mathrm{C}$ for more than 1.5 hour (In Figure 6(b)). After phosphor slurry converted into solid, we removed the sample from the mold and mounted it using inlay material as Figure 6(c) shows. Finally, Longitudinal cross-sectional microstructure photograph of the sample was acquired using scanning electron microscope (SEM), as seen in Figure 7. It is confirmed that phosphor sedimentation occurs obviously during isothermal curing at $85^{\circ} \mathrm{C}$. Besides, Figure $6(\mathrm{~d})$ shows the longitudinal

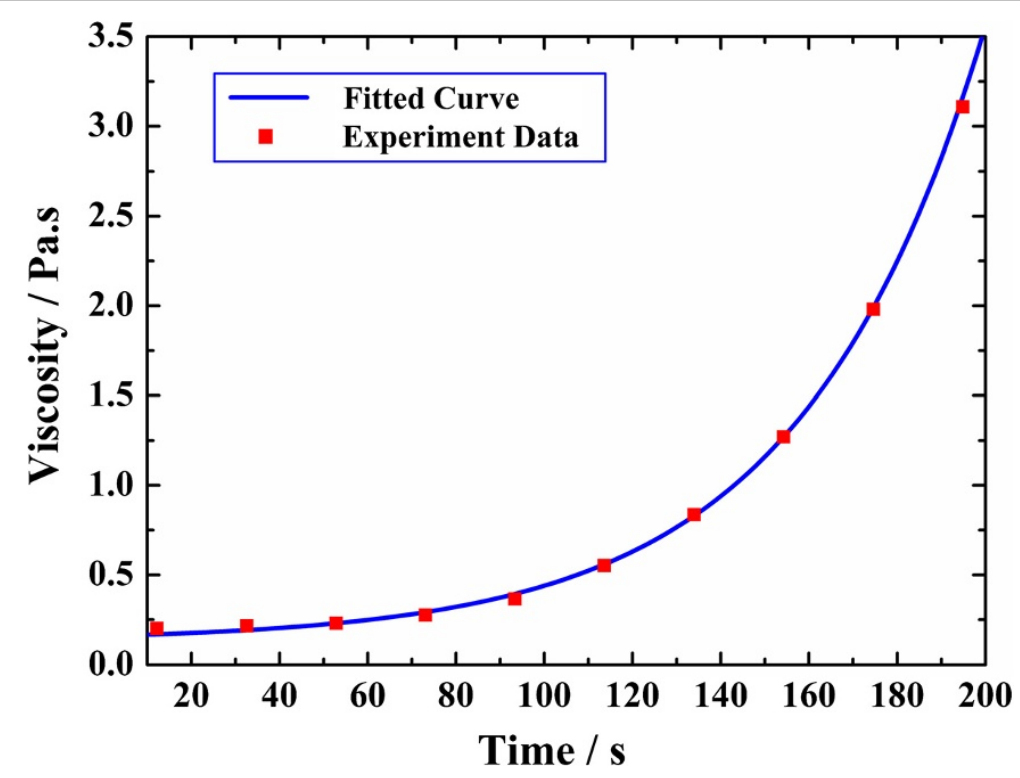

Figure 5 Relationship between the viscosity of silicone and time during isothermal curing at $85^{\circ} \mathrm{C}$. 


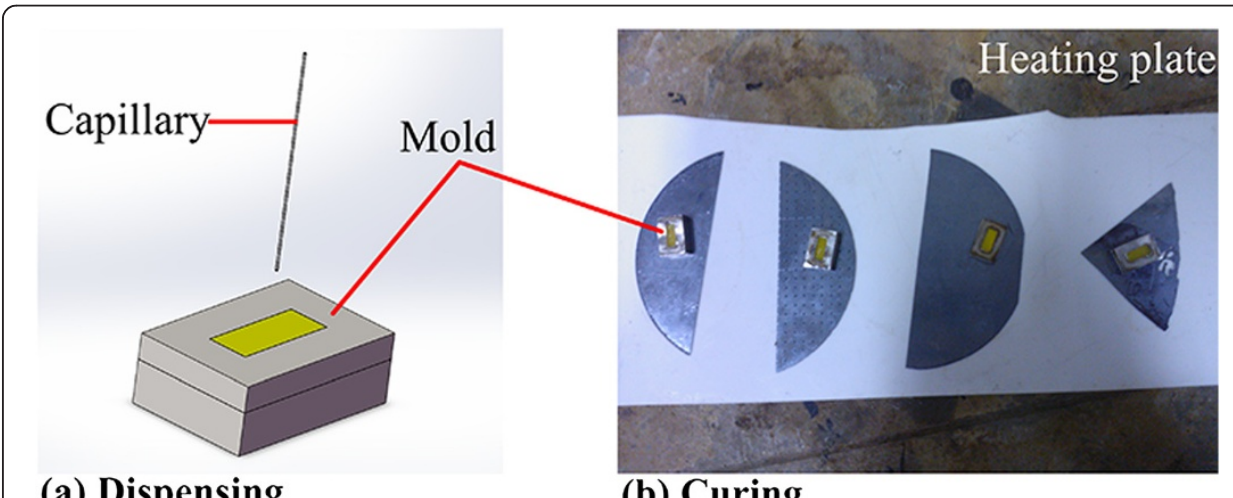

(a) Dispensing

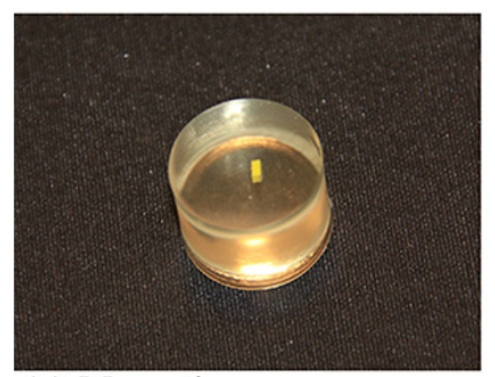

(c) Mounting (b) Curing

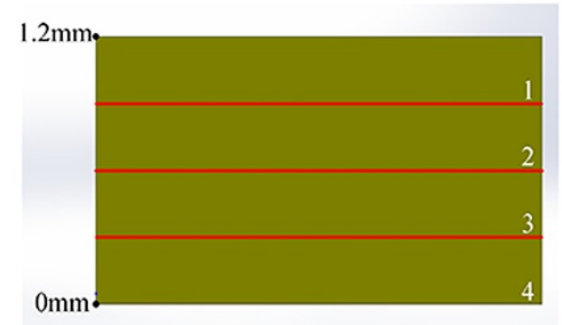

(d) Longitudinal section of the sample

Figure 6 Processes of the experiment. (a) Dispensing (b) Curing (c) Mounting (d) Longitudinal section of the sample.

cross-section of the sample. For purposes of analysis, the cross-section is quartered as the red lines. This part will be further explained later.

\section{Results and disussions}

Modeling analysis

The simulation result is shown in Figure 8, whose subgraphs show the distribution of the phosphor particles at the beginning, after $1 \mathrm{~min}, 4 \mathrm{~min}, 5 \mathrm{~min}, 6 \mathrm{~min}$ and $1.5 \mathrm{~h}$ respectively. As Figure 8 shows, phosphor particles distribute uniformly in the silicone at the beginning, and sedimentation phenomenon occurs with the lapse of time. However, the distribution of the phosphor particles in Figure 8(d) to (f) are extremely the same, and subtly different from the distribution in Figure 8(c), which means the sedimentation of the phosphor particles mostly happens in the first 5 minutes during isothermal curing at $85^{\circ} \mathrm{C}$. Besides, from Figure 8 we can see that at different time during curing, particles in the bottom share bigger average size than those apart from the bottom, which is largely because that the sedimentation velocity is proportional to the size of the particle.

Figure 9 shows the sedimentation of the particle whose diameter is $13 \mu \mathrm{m}$. The solid line represents the velocity of the particle at any time during the sedimentation, and the dots represent the position of the particle. As we can see, the velocity gradually decreases with the lapse of time due to the increasing viscosity of the silicone. What's more, the velocity is close to zero after about five minutes. The displacement of the particle during the sedimentation is $0.188 \mathrm{~mm}$ which accounts for $15.67 \%$ of the total thickness. 


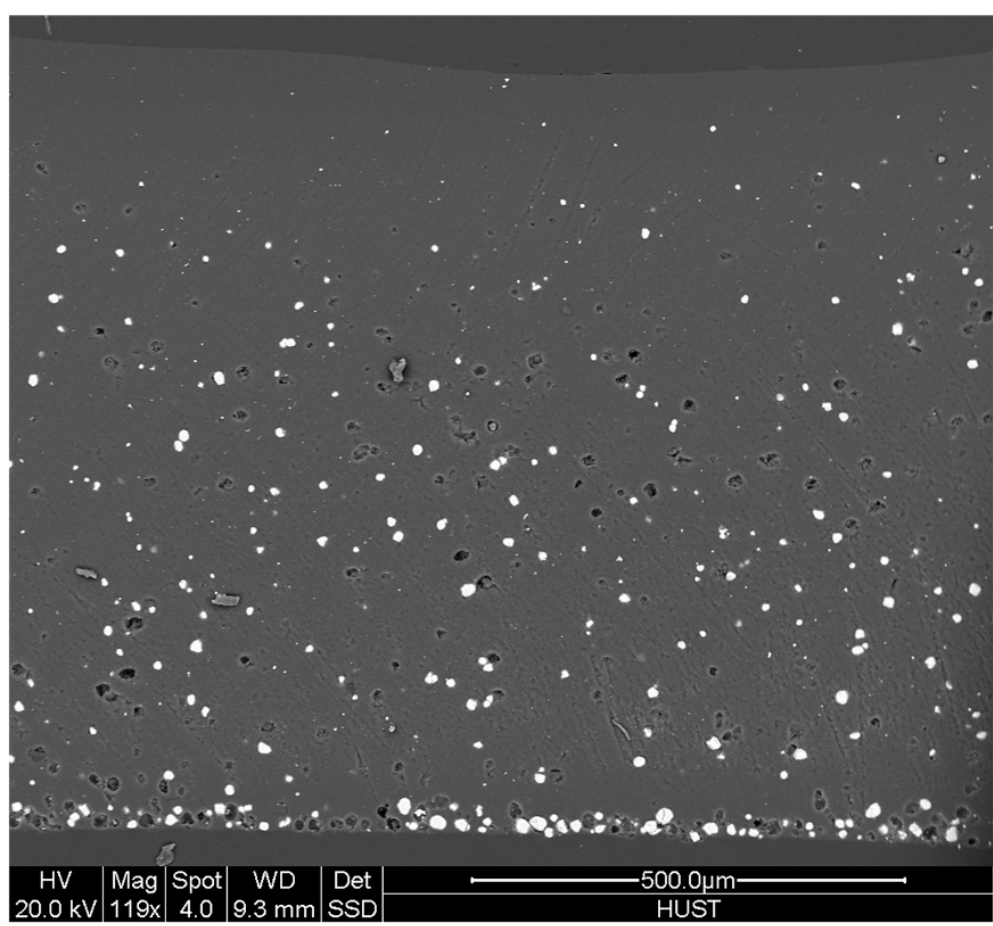

Figure 7 Longitudinal cross-sectional microstructure photograph of sample.

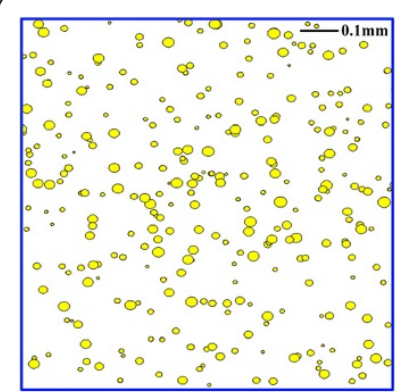

(a) $\mathrm{t}=0$

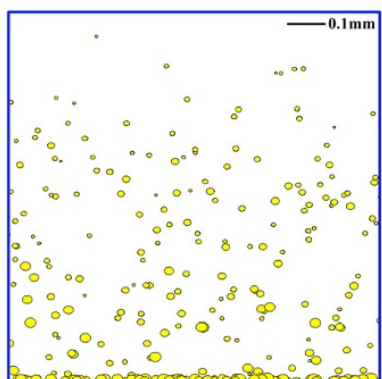

(d) $\mathrm{t}=5 \mathrm{~min}$

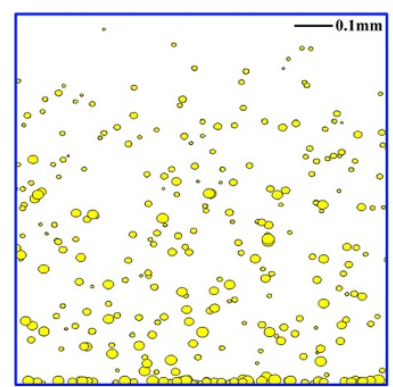

(b) $\mathrm{t}=1 \mathrm{~min}$

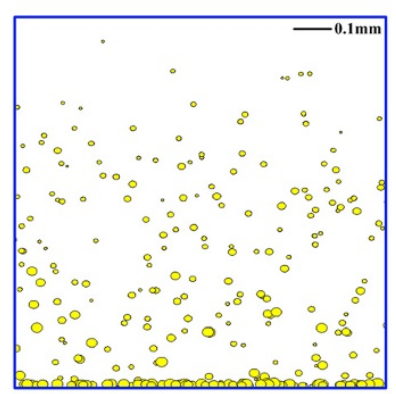

(e) $\mathrm{t}=6 \mathrm{~min}$

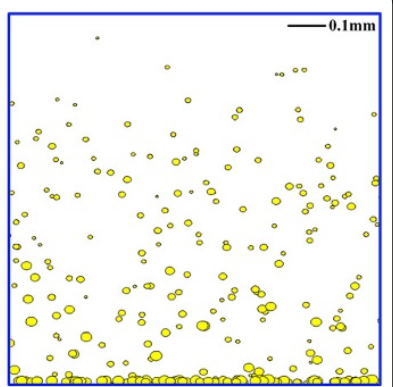

(c) $\mathrm{t}=4 \mathrm{~min}$

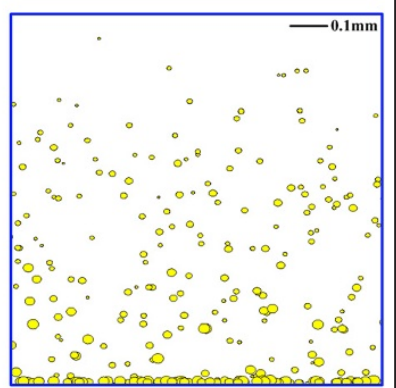

(f) $\mathrm{t}=1.5 \mathrm{~h}$

Figure 8 Simulation result (the distribution of phosphor particles). (a) at the beginning (b) $t=1$ min (c) $t=4 \min (\mathbf{d}) t=5 \min (\mathbf{e}) \mathrm{t}=6 \min$ (f) $t=1.5 \mathrm{~h}$. 


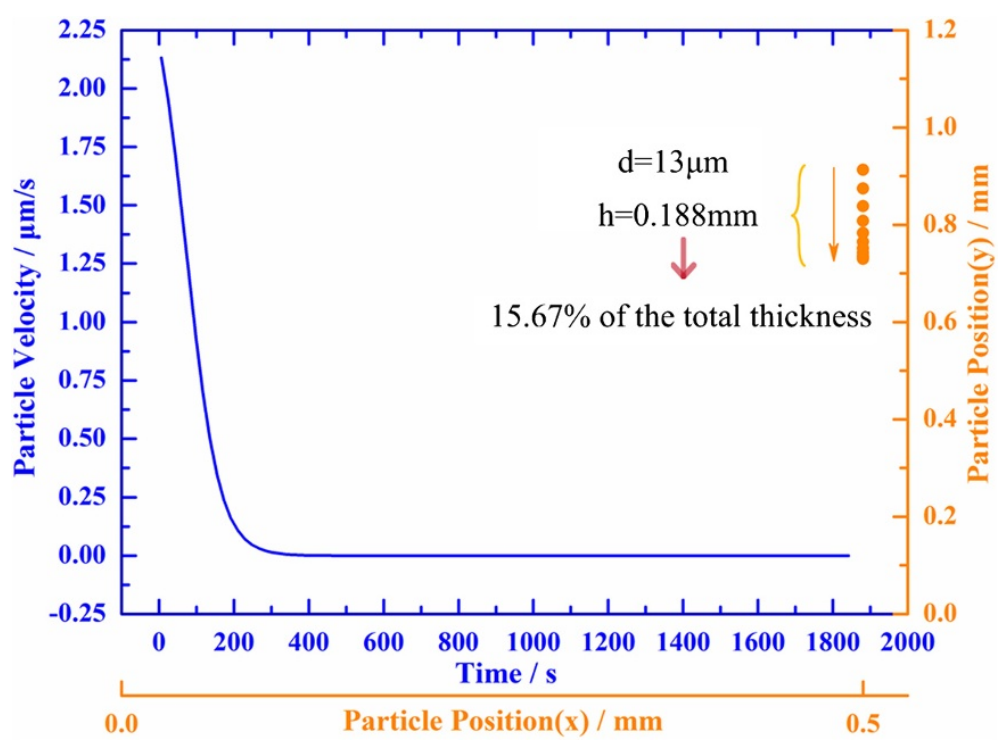

Figure 9 Sedimentation velocity of the particle $(d=13 \mu \mathrm{m})$ at any time during the sedimentation.

\section{Comparison with experiment}

Quartering Figure 7 and Figure 8(f) as the red lines, we can get Figure 10. Figure 10 shows the comparison between simulation and experiment results. In Figure 10, abscissa (Number) means the number of the section, and ordinate (Area fraction) means the particles' area in each section to the total area ratio. Here, we can see that the area fractions in experiment and simulation results correspond to equal. In other words, concentration gradients of phosphor in experiment and simulation results are nearly the same. Thus, the simulation result shows good agreement with the experimental result. Therefore, the model can well simulate the sedimentation of phosphor particle in silicone during isothermal curing.

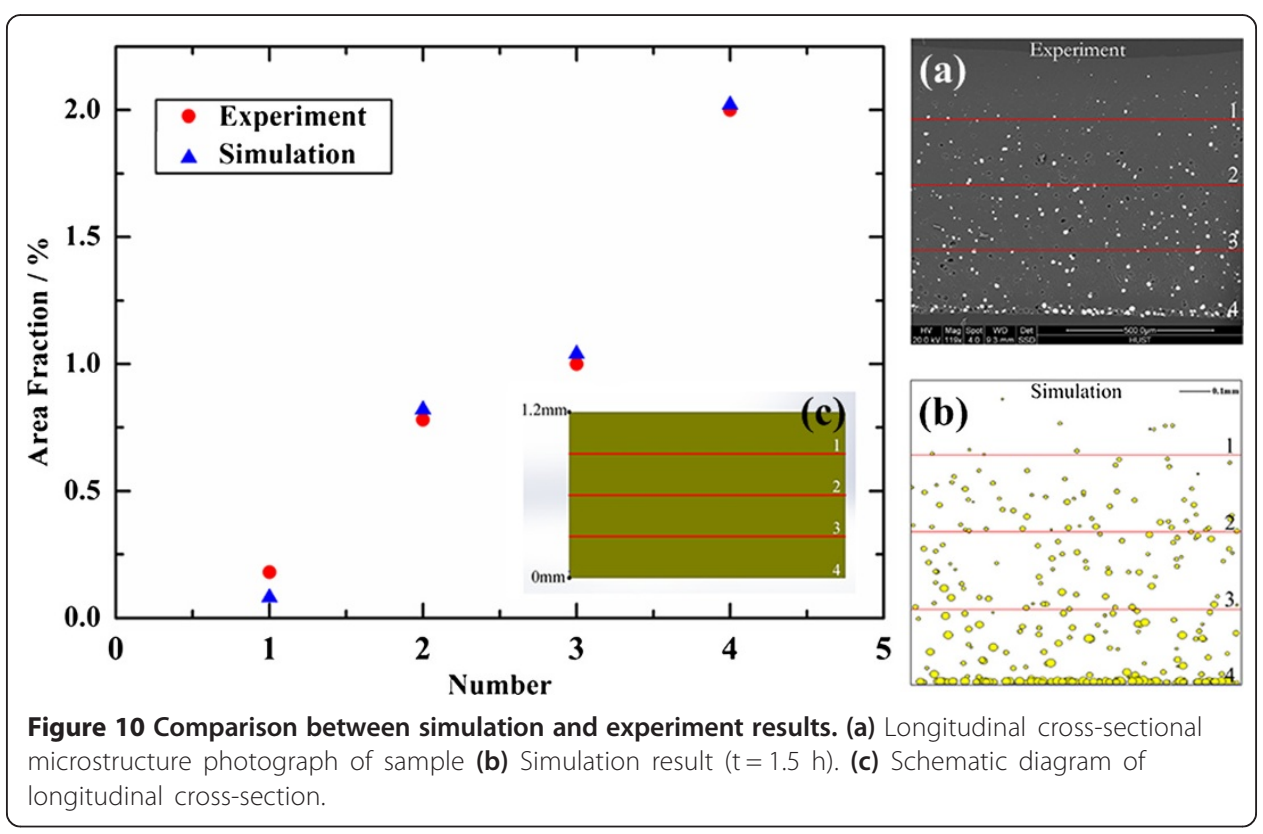




\section{Conclusions}

According to the Stokes' law, a model was developed to investigate phosphor sedimentation in silicone during isothermal curing. Experiment was conducted and the comparison between the experiment and simulation results shows that the model can well simulate the sedimentation of the phosphor particles in silicone during isothermal curing.

The results indicate that the sedimentation velocity is inversely proportional to the viscosity of silicone and proportional to the size of phosphor particles. During isothermal curing, the resistance force $\left(F_{\text {Stokes }}=6 \pi \eta r v\right)$ acting on the phosphor particle increases with the increasing viscosity as time goes by. The velocity of the phosphor particle $(\mathrm{d}=13 \mu \mathrm{m})$ is close to zero after about five minutes, and the displacement is $0.188 \mathrm{~mm}$ which accounts for $15.67 \%$ of the total thickness. Therefore, phosphor sedimentation occurs obviously during isothermal curing at $85^{\circ} \mathrm{C}$. The way to suppress the occurrence of phosphor sedimentation is now under investigation. We suppose to find a curing condition in which the distribution can be more uniform using our sedimentation model in further study.

Competing interests

The authors declare that they have no competing interests.

\section{Authors' contributions}

YW carried out the programming and experiments as the first author. $\mathrm{HZ}$ provided guidance for the experiments and participated in the result analysis. $\mathrm{RH}$ gave advice on the modeling method. XB instructed the overall work as the supervisor. All authors read and approved the final manuscript.

\section{Acknowledgments}

The authors would like to acknowledge the financial support in part by National Natural Science Foundation of China (51376070) and in part by 973 Project of The Ministry of Science and Technology of China (2011CB013105).

Received: 16 October 2013 Accepted: 23 December 2013

Published: 23 April 2014

References

1. Steigerwald DA, Bhat JC, Collins D, Fletcher RM, Holcomb MO, Ludowise MJ, Martin PS, Rudaz SL: Illumination with solid state lighting technology. IEEE J Sel Top Quant Electron 2002, 8(2):310-320.

2. Pimputkar S, Speck JS, DenBaars SP, Nakamura S: Prospects for LED lighting. Nat Photon 2009, 3:180-182.

3. Hu R, Luo XB, Zheng H, Qin Z, Gan ZQ, Wu BL, Liu S: Design of a novel freeform lens for LED uniform illumination and conformal phosphor coating. Optics Express 2012, 20(13):13727-13737.

4. Liu S, Luo XB: LED Packaging for Lighting Applications: Design, Manufacturing, and Testing Singapore: John Wiley \& Sons; 2011.

5. Sommer C, Reil F, Krenn JR, Hartmann P, Pachler P, Hoschopf H, Wenzl FP: The impact of light scattering on the radiant flux of phosphor-converted high power white light-emitting diodes. J Lightwave Tech 2011, 29(15):2287-2291.

6. Lee KC, Kim DG, Kim SM, Oh HS, Baek JH: The Influence of Phosphor Sedimentation on the White LEDswith Different Structure Chip. SPIE 2011, 8312:83120E.

7. Hu R, Wang YM, Zou Y, Chen X, Liu S, Luo XB: Study on phosphor sedimentation effect in white light-emitting diodepackages by modeling multi-layer phosphors with the modified Kubelka-Munk theory. J Appl Phys 2013, 113:063108.

8. Choi C, Yatsuzuka K, Asano K: Dynamic Motion of a Conductive Particle in ViscousFluid Under DC Electric Field. IEEE Trans Ind App/ 2001, 37(3):785-791.

9. Kiuna N, Lawrence CJ, Fontana QPV, Lee PD, Selerland T, Spelt PDM: A model for resin viscosity during cure in the resintransfer moulding process. Composites A 2002, 33:1497-1503. 